\title{
INDÚSTRIA TÊXTIL DE SANTA CATARINA E SUA CAPACIDADE INOVADORA: ESTUDO SOB A PERSPECTIVA DA EFICIÊNCIA, EFICÁCIA, CUSTOS E MELHORIA DE PROCESSOS
}

\author{
Giancarlo Gomes \\ Doutor em Ciências Contábeis e Administração pela Fundação Universidade Regional de Blumenau - \\ FURB \\ Professor da Fundação Universidade Regional de Blumenau - FURB \\ giancarlo@pzo.com.br (Brasil)
}

\author{
Denise Del Prá Netto Machado \\ Doutora em Administração de Empresas pela Fundação Getúlio Vargas - FGV/SP \\ Professora da Fundação Universidade Regional de Blumenau - FURB \\ profadenisedelpra@gmail.com (Brasil)
}

\section{Joaquin Alegre}

Doutor em Administração e Direção de Empresas - Universidad Jaume I - Espanha

Professor Titular do Dpto. de Direção de Empresas da Universidade de Valencia - Espanha joaquin.alegre@uv.es (Espanha)

\section{RESUMO}

Santa Catarina se destaca como um dos principais parques produtivos do setor têxtil. Assim como as demais indústrias têxteis brasileiras, a catarinense vem sofrendo pressões de mercado, principalmente dos países asiáticos. O estudo teve como objetivo identificar se as dimensões - eficiência, eficácia, custos e as melhorias de processo - estão associadas ao desempenho da inovação na indústria têxtil de Santa Catarina. A pesquisa foi descritiva, exploratória e causal, de levantamento ou survey $e$ quantitativa. A amostra foi composta por 441 respondentes de 16 organizações do estado de Santa Catarina. Os dados foram analisados por meio de análise fatorial exploratória, análise fatorial confirmatória e modelagem de equações estruturais. Os resultados apontam que o desempenho na inovação de produtos pode ser abordado utilizando as dimensões de primeira ordem eficácia, eficiência, melhorias no processo de produção e redução de custos. Os resultados foram maiores na dimensão melhorias, refletindo maior atenção no aprimoramento da qualidade do produto, nas condições de trabalho e na flexibilidade produtiva. O tamanho da organização não teve efeito significativo no desempenho em inovação de produtos nas organizações estudadas. Entende-se que os resultados alcançados pelo presente estudo possam ser considerados relevantes tanto por seu impacto sobre a teoria quanto por sua aplicação gerencial. Pesquisas futuras devem ser direcionadas no sentido de analisar se em outros setores existem diferenças nos constructos do desempenho em inovação de produtos em decorrência de características particulares de cada setor.

Palavras-chave: Capacidade Inovadora; Inovação em Produtos e Processos; Indústria Têxtil. 


\section{INTRODUÇÃO}

A inovação se converteu em um fator significativo no momento de determinar os resultados empresariais. As contribuições da inovação em relação aos resultados estratégicos, e, portanto, à sobrevivência em longo prazo, têm sido amplamente reconhecidas e discutidas na literatura (Alegre \& Chiva, 2008). A importância do tema é evidenciada por autores que vêm estudando o assunto, tornando seu conceito mais extenso e complexo (Van De Ven et al., 1999; Damanpour, 1991; Afuah, 2003; Tidd, Bessant \& Pavitt, 2005). Esta realidade também pertence às indústrias com menor valor agregado, como as têxteis que se utilizam da moda para aumentar a velocidade do ciclo do produto no mercado.

A inovação é um fenômeno inerente e fundamental para a prosperidade da indústria têxtil. A tendência de mudanças rápidas na moda, conjugada à diferenciação do produto, é crucial para impedir a invasão de produtos asiáticos. Isto se deve a velocidade das mudanças e a distância física dos centros de consumo, que dificultam a chegada desses produtos em tempo hábil para que ainda estejam na moda (Costa \& Rocha, 2009; Monteiro \& Veiga, 2009). A base do sistema de moda reside na ideia da mudança contínua, na obsolescência programada e na introdução de produtos que sejam percebidos como novos, mesmo que não sejam caracterizados como uma invenção. Produtos e serviços inovadores são criados com o objetivo de atender as necessidades encontradas no mercado para satisfazer necessidades de clientes (Rech, 2006).

Atuando em um mercado altamente competitivo, o Brasil tem perdido espaço no setor têxtil e de confecções. Mesmo com o crescimento do consumo mundial de têxteis e confeccionados, a participação do país no comércio mundial diminuiu nos últimos anos. Ao mesmo tempo, houve um acirramento da concorrência global, tendo em vista o crescimento exponencial dos produtos asiáticos no comércio internacional (Costa \& Rocha, 2009).

No cenário nacional, o estado de Santa Catarina se destaca como um dos principais parques produtivos do setor têxtil. Assim como as demais indústrias têxteis brasileiras, a catarinense vem sofrendo pressões mercadológicas, principalmente dos países asiáticos. O setor pode ser considerado um dos mais atingidos pelo processo de abertura comercial, estando em um momento de crise, em função do grande aumento das importações, sobretudo no segmento de fios e tecidos artificiais e sintéticos provenientes de outros países (Mendes, 2003).

Assim como o conjunto da indústria têxtil e de confecções nacional, as organizações sediadas no estado de Santa Catarina desenvolveram estratégias para acompanhar esse processo de mudança,

Revista de Administração e Inovação, São Paulo, v. 11, n.2, p. 273-294, abr./jun. 2014. 
tanto sob a perspectiva interna em relação à abertura econômica, quanto no que se refere às transformações no padrão de concorrência internacional do setor (Fernandes \& Cario, 2009). Nessa conjuntura, tornou-se fundamental para a sobrevivência das indústrias têxteis desenvolverem estratégias competitivas diferenciadas, baseadas na utilização da inovação como um instrumento relevante para a inserção no mercado mundial (Costa \& Rocha, 2009).

As transformações tecnológicas trouxeram implicações sobre a competição na indústria têxtil, principalmente à delimitação de novos espaços da concorrência mais internacionalizados e a aceleração do ritmo de inovação tecnológica com encurtamento do ciclo de vida de produtos e processos e aumento da diferenciação de produtos. No novo paradigma competitivo predomina qualidade de produto, flexibilidade, rapidez de entrega e inovatividade, além da racionalização dos custos de produção (Rech, 2006). Diante dessa problemática, o estudo tem como objetivo identificar se as dimensões - eficiência, eficácia, custos e as melhorias de processo - estão associadas ao desempenho da inovação na indústria têxtil de Santa Catarina.

O artigo está estruturado da seguinte forma, primeiramente é apresentada uma contextualização teórica sobre desenvolvimento de novos produtos, e com base na literatura são descritas as hipóteses do estudo. Na sequência é exposto o método utilizado na pesquisa, seguido pela análise dos resultados e testes das hipóteses. Por fim, são apresentadas as considerações e contribuições do estudo.

\section{MODELO TEÓRICO E HIPÓTESES}

A inovação de produto ou processo tem sido frequentemente utilizada pelas organizações com o objetivo de obter diferenciais competitivos (Ettlie, 1983; Hull, Hage, \& Azumi, 1985; Zhuang \& Wiliamson; Carter, 1999). A inovação no processo, por sua vez, é compreendida como os elementos que são agregados ao processo de operações, adição de materiais, tarefas, instrumentos ou fluxo de determinados mecanismos na produção, na distribuição de produtos ou na prestação de serviços (Knight, 1967; Utterback \& Abernathy, 1975; Jonash \& Sommerlatte, 2001).

Produtos inovadores são produtos ou serviços criados com o objetivo de atender as necessidades encontradas no mercado para satisfazer necessidades de clientes (Knight, 1967; Utterback \& Abernathy, 1975; Jonash \& Sommerlatte, 2001). A inovação de produtos consiste na exploração bemsucedida de novas ideias (Alegre, Lapiedra, \& Chiva, 2006). Nesta pesquisa, a inovação do produto será abordada em quatro diferentes dimensões: eficácia, eficiência, melhorias no processo de produção e redução de custos.

A eficácia da inovação versa sobre os resultados econômicos da inovação de produtos, ou seja, sobre o impacto econômico da inovação na empresa, ou, ainda, sobre a importância econômica das 
saídas do processo de inovação. Assim, esta dimensão procura descobrir o que é obtido a partir da inovação (eficácia do resultado de inovação). A eficácia da inovação reflete o nível de sucesso da inovação (Ocde-Eurostat, 1997; Alegre, Lapiedra, \& Chiva, 2006; Bakar \& Ahmad, 2010; Henttonen, Ritala \& Jauhiainen, 2011). Com base no exposto é possível formular a seguinte hipótese: Hipótese 1: A Eficácia tem um efeito positivo no desempenho em inovação de produtos na indústria têxtil de Santa Catarina.

A segunda dimensão, por outro lado, se refere a recursos consumidos para atingir esses resultados, ou seja, do próprio processo. Ela se concentra em como realizar esse processo de inovação (a eficiência do processo). A eficiência da inovação reflete o esforço feito para alcançar determinado nível de sucesso (Wheelwright \& Clark, 1992; Ocde-Eurostat, 1997; Griffin, 1997; Zhan \& Doll, 2001; Valle \& Avella, 2003). A eficiência da inovação de produto é determinada pelo tempo do projeto de inovação. Além da relevância do tempo para determinar a eficiência do processo de inovação, alguns estudos têm também incluído uma avaliação subjetiva sobre a eficiência global de inovação do projeto (Wheelwright \& Clark, 1992; Alegre, Lapiedra, \& Chiva, 2006; Henttonen, Ritala, \& Jauhiainen, 2011). Assim, a seguinte hipótese pode ser formulada: Hipótese 2: A Eficiência tem um efeito positivo no desempenho em inovação de produtos na indústria têxtil de Santa Catarina.

Um dos desafios do setor têxtil é a obtenção da flexibilidade produtiva, com ganho de eficiência e produtividade, uma vez que o setor opera com uma diversidade de produtos com ciclo de vida extremamente reduzido por causa das tendências de moda (Rech, 2006). Fatores associados a uma maior flexibilidade, fundamentais para alcançar a inovação - como criatividade, autonomia e propensão ao risco - são difíceis de ser maximizados quando a organização coloca ênfase na estabilidade e no controle (Naranjo-Valencia, Jiménez, \& Sanz-Valle, 2012).

Os motivos de melhorias pelos quais uma organização se engaja em atividades de inovação podem ser identificados por meio de seus objetivos econômicos em termos de produtos e mercados e do modo como ela classifica uma série de metas que o processo de inovação pode colocar a seu alcance. Entre outros objetivos destacam-se: a redução dos danos ao meio ambiente; melhoraria na qualidade do produto; melhoraria nas condições de trabalho; e melhoraria na flexibilidade produtiva tal qual sugere a OCDE (2005). Isso leva à seguinte hipótese: Hipótese 3: As melhorias no processo de inovação têm um efeito positivo no desempenho em inovação de produtos na indústria têxtil de Santa Catarina.

Custos no desenvolvimento de novos produtos têm sido mensurados, tanto objetiva (Hoopes \& Postrel, 1999; Griffin, 1993; Pisano, 1994) quanto subjetivamente (Griffin \& Page, 1993; Griffin, 1997; Zhan \& Doll, 2001; Mcevily \& Chakravarthy, 2002; Valle \& Avella, 2003). Medidas objetivas 
dos custos de desenvolvimento de novos produtos usualmente se referem a um projeto de inovação específico que foi analisado em detalhes, enquanto medidas subjetivas têm sido geralmente aplicadas em pesquisas sobre inovação (Alegre, Lapiedra, \& Chiva, 2006).

Os custos decorrentes das inovações tecnológicas podem ser diminuídos por meio da redução dos custos unitários de mão de obra; do corte de materiais de consumo; do corte do consumo de energia; da redução da taxa de rejeição; da redução dos custos de desenho do produto; da redução dos prazos de início de produção (OCDE, 2005). Desta forma, é possível formular a hipótese: Hipótese 4: A redução de custos no processo de inovação tem um efeito positivo no desempenho em inovação de produtos na indústria têxtil de Santa Catarina.

No que ser refere à influência do porte da organização na inovação, Damanpour (1996), salienta que o tamanho de uma organização é um dos fatores que interferem na sua estrutura e em seu processo de inovação. Grandes organizações apresentam algumas vantagens, como maior disponibilidade de recursos. Entretanto podem ser mais burocratizadas e menos flexíveis, sendo, desse modo, mais resistentes às mudanças.

A influência do porte da organização pode variar entre as indústrias também. Em alguns setores industriais, as organizações menores tendem a levar vantagem, como, por exemplo, as indústrias cujo desenvolvimento tecnológico demanda flexibilidade das atividades de P\&D e agilidade nas tomadas de decisões (Freeman \& Soete, 2008). Os resultados da meta-análise de Damanpour (1991) sugerem a existência de uma associação positiva entre o tamanho organizacional e o desempenho em inovação. Consequentemente, formula-se: Hipótese 5: O tamanho da organização - conforme critérios pelo número de empregados - tem efeito positivo no desempenho em inovação de produtos na indústria têxtil de Santa Catarina.

Os resultados econômicos da inovação de produtos podem ser obtidos por meio do método da autoavaliação pelos diretores, sobre as inovações desenvolvidas por suas empresas. A autoavaliação sobre o número de inovações introduzidas pelas empresas já é largamente utilizada nos países da OCDE. Ela consiste em perguntar diretamente aos diretores e colaboradores pelas inovações tecnológicas desenvolvidas durante determinado período. Esse método oferece, em primeira mão, informações sobre o resultado do processo de inovação (Kleinknecht, 1993, Coombs \& Tomlinson, 1998).

O Manual de Oslo da Organização para a Cooperação e Desenvolvimento Econômico (OCDE) oferece uma escala de medida para a avaliação detalhada dos objetivos econômicos da inovação (Ocde-Eurostat, 1997). Essa escala foi apresentada pela OCDE para fornecer alguns controladores coerentes para estudos sobre inovação, conseguindo assim uma maior homogeneidade e 
comparabilidade entre os estudos (Alegre, Lapiedra, \& Chiva, 2006; Alegre, Chiva \& Lapiedra, 2009; Bakar \& Ahmad, 2010; Henttonen, Ritala, \& Jauhiainen, 2011).

Em 2001, uma iniciativa da Red Iberoamericana de Indicadores de Ciencia y Tecnología (RICYT), da Organización de Estados Americanos (OEA) e do Programa Iberoamericano de Ciencia y Tecnología para el Desarrollo (CYTED) desenvolveu o Manual de Bogotá, que, com base no Manual de Oslo, visando garantir a comparabilidade internacional, sugere algumas referências para a adequação dos indicadores de inovação às especificidades que caracterizam os sistemas de inovação e as empresas da América Latina e Caribe. Com base nisso, para a formulação do instrumento de coleta de dados foram levadas em consideração as especificidades das organizações brasileiras.

\section{MÉTODOS E TÉCNICAS DE PESQUISA}

O método empregado foi exploratório, causal e quantitativo. No caso específico deste estudo, a pesquisa caracteriza-se como exploratória, em face de ser reduzido o número de estudos sobre desenvolvimento de inovações de produtos sob a perspectiva dos indicadores apresentados nesse estudo - eficiência, eficácia, custos e as melhorias de processo. Poucas pesquisas foram realizadas sobre o tema em organizações brasileiras, particularmente em indústrias têxteis. Neste estudo não se buscou somente expor fatos, e sim compreender a relação entre eles, ou seja, a relação causal entre as dimensões do desempenho na inovação de produtos. Os estudos causais visam descobrir o efeito que uma variável tem sobre outra (ou outras) ou sobre certos resultados obtidos (Cooper \& Schindler, 2003).

A análise de uma única indústria pode ser vantajosa para a análise da inovação, uma vez que ela se manifesta de várias maneiras em diferentes indústrias. O setor têxtil e de confecção brasileiro tem destaque no cenário mundial, e o estado de Santa Catarina têm o setor têxtil como grande gerador de empregos e como característica o predomínio de empresas de micro e pequeno porte na confecção de malhas, ao lado de grandes empresas líderes no setor (SEBRAE, 2012).

O constructo da pesquisa está dividido em quatro blocos: no primeiro bloco está descrito variáveis e subvariáveis do constructo referente a dimensão Eficácia da inovação; no segundo estão descritas as variáveis e subvariáveis da dimensão Eficiência; no terceiro bloco, as categorias da dimensão Melhorias; e, no último, o constructo da pesquisa e as variáveis e subvariáveis da dimensão Custos. O constructo da pesquisa referente ao desempenho em inovação de produtos é apresentado no Quadro 1.

Revista de Administração e Inovação, São Paulo, v. 11, n.2, p. 273-294, abr./jun. 2014. 
Quadro 1 - Constructo do Desempenho em inovação de produtos

\begin{tabular}{|c|c|c|}
\hline Fator/Variável & Marco Teórico & Dimensão \\
\hline $\begin{array}{l}\text { Substituição de produtos } \\
\text { ultrapassados (Eficacia1). }\end{array}$ & $\begin{array}{l}\text { Ocde-Eurostat (1997); Alegre, Lapiedra e Chiva (2006); Alegre e } \\
\text { Chiva (2008); Alegre, Chiva e Lapiedra (2009); Bakar e Ahmad } \\
\text { (2010); Henttonen, Ritala e Jauhiainen (2011). }\end{array}$ & \multirow{7}{*}{ Eficácia } \\
\hline $\begin{array}{l}\text { Ampliação da linha de } \\
\text { produtos (Eficacia2). }\end{array}$ & $\begin{array}{l}\text { Ocde-Eurostat (1997); Alegre, Lapiedra e Chiva (2006); Alegre e } \\
\text { Chiva (2008); Alegre, Chiva e Lapiedra (2009); Bakar e Ahmad } \\
\text { (2010); Henttonen, Ritala e Jauhiainen (2011). }\end{array}$ & \\
\hline $\begin{array}{l}\text { Desenvolvimento de produtos } \\
\text { fora do segmento principal da } \\
\text { organização (produtos } \\
\text { secundários) (Eficacia3) }\end{array}$ & $\begin{array}{l}\text { Ocde-Eurostat (1997); Alegre, Lapiedra e Chiva (2006); Alegre e } \\
\text { Chiva (2008); Alegre, Chiva e Lapiedra (2009); Bakar e Ahmad } \\
\text { (2010); Henttonen, Ritala e Jauhiainen (2011). }\end{array}$ & \\
\hline $\begin{array}{l}\text { Desenvolvimento de novas } \\
\text { linhas de produtos (Eficacia4). }\end{array}$ & $\begin{array}{l}\text { Ocde-Eurostat (1997); Alegre, Lapiedra e Chiva (2006); Alegre e } \\
\text { Chiva (2008); Alegre, Chiva e Lapiedra (2009); Bakar e Ahmad } \\
\text { (2010); Henttonen, Ritala e Jauhiainen (2011). }\end{array}$ & \\
\hline $\begin{array}{l}\text { Desenvolvimento de produtos } \\
\text { ecológicos (Eficacia5). }\end{array}$ & $\begin{array}{l}\text { Ocde-Eurostat (1997); Alegre, Lapiedra e Chiva (2006); Alegre e } \\
\text { Chiva (2008); Alegre, Chiva e Lapiedra (2009); Bakar e Ahmad } \\
\text { (2010); Henttonen, Ritala e Jauhiainen (2011). }\end{array}$ & \\
\hline $\begin{array}{l}\text { Aumento da participação de } \\
\text { mercado (Eficacia6). }\end{array}$ & $\begin{array}{l}\text { Ocde-Eurostat (1997); Alegre, Lapiedra e Chiva (2006); Alegre e } \\
\text { Chiva (2008); Alegre, Chiva e Lapiedra (2009); Bakar e Ahmad } \\
\text { (2010); Henttonen, Ritala e Jauhiainen (2011). }\end{array}$ & \\
\hline $\begin{array}{l}\text { Abertura de novos mercados } \\
\text { nacionais (Eficacia7). }\end{array}$ & $\begin{array}{l}\text { Ocde-Eurostat (1997); Alegre, Lapiedra e Chiva (2006); Alegre e } \\
\text { Chiva (2008); Alegre, Chiva e Lapiedra (2009); Bakar e Ahmad } \\
\text { (2010); Henttonen, Ritala e Jauhiainen (2011). }\end{array}$ & \\
\hline $\begin{array}{l}\text { Tempo médio em semanas } \\
\text { para o desenvolvimento do } \\
\text { produto (Eficienci1) }\end{array}$ & $\begin{array}{l}\text { Gupta e Wilemon (1990); Mabert, Muth e Schmenner (1992); } \\
\text { Cooper e Kleinschmidt, (1993); Wheelwright e Clark (1992); Pisano } \\
\text { (1994); Chiesa, Coughlan e Voss (1996); Ocde-Eurostat (1997); } \\
\text { Alegre, Lapiedra e Chiva (2006); Alegre e Chiva (2008); Alegre, } \\
\text { Chiva e Lapiedra (2009); Bakar e Ahmad (2010); Henttonen, Ritala } \\
\text { e Jauhiainen (2011). }\end{array}$ & \multirow{4}{*}{ Eficiência } \\
\hline $\begin{array}{l}\text { Tempo médio em horas totais } \\
\text { desenvolvimento do produto } \\
\text { (Eficienci2). }\end{array}$ & $\begin{array}{l}\text { Gupta e Wilemon (1990); Mabert, Muth e Schmenner (1992); } \\
\text { Cooper e Kleinschmidt, (1993); Wheelwright e Clark (1992); Pisano } \\
\text { (1994); Chiesa, Coughlan e Voss (1996); Ocde-Eurostat (1997); } \\
\text { Alegre, Lapiedra e Chiva (2006); Alegre e Chiva (2008); Alegre, } \\
\text { Chiva e Lapiedra (2009); Bakar e Ahmad (2010); Henttonen, Ritala } \\
\text { e Jauhiainen (2011). }\end{array}$ & \\
\hline $\begin{array}{l}\text { Custo médio por projeto de } \\
\text { inovação (Eficienci3). }\end{array}$ & $\begin{array}{l}\text { Gupta e Wilemon (1990); Wheelwright e Clark (1992); Pisano } \\
\text { (1994); Chiesa, Coughlan e Voss (1996); Ocde-Eurostat (1997); } \\
\text { Alegre, Lapiedra e Chiva (2006); Alegre e Chiva (2008); Alegre, } \\
\text { Chiva e Lapiedra (2009); Bakar e Ahmad (2010); Henttonen, Ritala } \\
\text { e Jauhiainen (2011). }\end{array}$ & \\
\hline $\begin{array}{l}\text { Grau de satisfação com o } \\
\text { produto (Eficienci4). }\end{array}$ & $\begin{array}{l}\text { Ancona e Caldwell (1990); Ancona e Caldwell (1992); Chiesa, } \\
\text { Coughlan e Voss (1996); Alegre, Lapiedra e Chiva (2006); Alegre e } \\
\text { Chiva (2008); Alegre, Chiva e Lapiedra (2009); Bakar e Ahmad } \\
\text { (2010); Henttonen, Ritala e Jauhiainen (2011). }\end{array}$ & \\
\hline $\begin{array}{l}\text { Redução dos danos ao meio } \\
\text { ambiente (Melhora1). }\end{array}$ & $\begin{array}{l}\text { Ocde-Eurostat (1997); Alegre, Lapiedra e Chiva (2006); Alegre e } \\
\text { Chiva (2008); Alegre, Chiva e Lapiedra (2009); Bakar e Ahmad } \\
\text { (2010); Henttonen, Ritala e Jauhiainen (2011). }\end{array}$ & \multirow{4}{*}{ Melhorias } \\
\hline $\begin{array}{l}\text { Melhoria das condições de } \\
\text { trabalho (Melhora2). }\end{array}$ & $\begin{array}{l}\text { Ocde-Eurostat (1997); Alegre, Lapiedra e Chiva (2006); Alegre e } \\
\text { Chiva (2008); Alegre, Chiva e Lapiedra (2009); Bakar e Ahmad } \\
\text { (2010); Henttonen, Ritala e Jauhiainen (2011). }\end{array}$ & \\
\hline $\begin{array}{l}\text { Melhoria da qualidade do } \\
\text { produto (Melhora3). }\end{array}$ & $\begin{array}{l}\text { Ocde-Eurostat (1997); Alegre, Lapiedra e Chiva (2006); Alegre e } \\
\text { Chiva (2008); Alegre, Chiva e Lapiedra (2009); Bakar e Ahmad } \\
\text { (2010); Henttonen, Ritala e Jauhiainen (2011). }\end{array}$ & \\
\hline $\begin{array}{l}\text { Melhoria da flexibilidade } \\
\text { produtiva (Melhora4). }\end{array}$ & $\begin{array}{l}\text { Ocde-Eurostat (1997); Alegre, Lapiedra e Chiva (2006); Alegre e } \\
\text { Chiva (2008); Alegre, Chiva e Lapiedra (2009); Bakar e Ahmad } \\
\text { (2010); Henttonen, Ritala e Jauhiainen (2011). }\end{array}$ & \\
\hline
\end{tabular}


Continuação...

\begin{tabular}{|l|l|l|}
\hline $\begin{array}{l}\text { Redução dos custos de } \\
\text { produção por meio da } \\
\text { diminuição do ciclo de } \\
\text { fabricação (Custos1). }\end{array}$ & $\begin{array}{l}\text { Ocde-Eurostat (1997); Alegre, Lapiedra e Chiva (2006); Alegre e } \\
\text { Chiva (2008); Alegre, Chiva e Lapiedra (2009); Bakar e Ahmad } \\
\text { (2010); Henttonen, Ritala e Jauhiainen (2011). }\end{array}$ & \\
\hline $\begin{array}{l}\text { Redução dos custos de } \\
\text { produção por meio da } \\
\text { diminuição dos custos do } \\
\text { projeto. (Custos2). }\end{array}$ & $\begin{array}{l}\text { Ocde-Eurostat (1997); Alegre, Lapiedra e Chiva (2006); Alegre e } \\
\text { Chiva (2008); Alegre, Chiva e Lapiedra (2009); Bakar e Ahmad } \\
\text { (2010); Henttonen, Ritala e Jauhiainen (2011). }\end{array}$ & Custos \\
$\begin{array}{l}\text { Redução dos custos de } \\
\text { produção por meio da redução } \\
\text { da taxa de produção de } \\
\text { rejeitados (Custos3) }\end{array}$ & $\begin{array}{l}\text { Ocde-Eurostat (1997); Alegre, Lapiedra e Chiva (2006); Alegre e } \\
\text { Chiva (2008); Alegre, Chiva e Lapiedra (2009); Bakar e Ahmad } \\
\text { (2010); Henttonen, Ritala e Jauhiainen (2011). }\end{array}$ & \\
\hline $\begin{array}{l}\text { Redução dos custos de } \\
\text { produção por meio de cortes } \\
\text { no consumo de energia } \\
\text { (Custos4). }\end{array}$ & $\begin{array}{l}\text { Ocde-Eurostat (1997); Alegre, Lapiedra e Chiva (2006); Alegre e } \\
\text { Chiva (2008); Alegre, Chiva e Lapiedra (2009); Bakar e Ahmad } \\
\text { (2010); Henttonen, Ritala e Jauhiainen (2011). }\end{array}$ & \\
\hline $\begin{array}{l}\text { Redução dos custos de } \\
\text { produção por meio de cortes } \\
\text { no consumo de materiais } \\
\text { (Custos5). }\end{array}$ & $\begin{array}{l}\text { Ocde-Eurostat (1997); Alegre, Lapiedra e Chiva (2006); Alegre e } \\
\text { Chiva (2008); Alegre, Chiva e Lapiedra (2009); Bakar e Ahmad } \\
\text { (2010); Henttonen, Ritala e Jauhiainen (2011). }\end{array}$ & \\
\hline $\begin{array}{l}\text { Diminuição dos custos de } \\
\text { produção mediante a redução } \\
\text { dos custos de trabalho por } \\
\text { unidades (Custos6). }\end{array}$ & $\begin{array}{l}\text { Ocde-Eurostat (1997); Alegre, Lapiedra e Chiva (2006); Alegre e } \\
\text { Chiva (2008); Alegre, Chiva e Lapiedra (2009); Bakar e Ahmad } \\
\text { (2010); Henttonen, Ritala e Jauhiainen (2011). }\end{array}$ & \\
\hline
\end{tabular}

Fonte: Elaboração própria.

O questionário foi composto de 21 questões, sendo sete para a dimensão eficácia, quatro para a dimensão eficiência, quatro para a dimensão melhorias no processo de produção e seis para a dimensão custos. As assertivas foram construídas por meio de escala Likert com pontuações variando de 1 a 7 , sendo 1 para "Muito pior do que os concorrentes" e 7 "Muito melhor que os concorrentes". Para análise dos dados utilizou-se análise descritiva, Análise Fatorial Confirmatória (AFC) e Modelagem de Equações Estruturais (MEE). A MEE pode ser vista como a combinação de dois métodos multivariados conhecidos: análise fatorial e análise de regressão múltipla (Hair Jr., et al., 2005). Nesse estudo foi utilizado o software $\operatorname{SPSS}^{\circledR}$ (Statistical Package for the Social Sciences) versão 19 e $\operatorname{AMOS}^{\circledR}$ versão 20.

A definição da amostra foi intencional, por acessibilidade ou conveniência tendo como base os objetivos da pesquisa. Portanto, buscou-se selecionar empresas que poderiam de alguma forma apresentar características que contribuam com a pesquisa a ser realizada. A amostra foi formada por 441 respondentes divididos em 16 empresas. Na definição dos respondentes, os empregados das empresas selecionadas, não se fez uso de formas aleatórias de seleção, houve uma escolha deliberada dos participantes por meio da técnica de amostragem não probabilística intencional, ficando a critério da organização participante a definição das pessoas que responderam ao questionário, porém solicitouse uma amostra de todos os níveis organizacionais.

Revista de Administração e Inovação, São Paulo, v. 11, n.2, p. 273-294, abr./jun. 2014. 
Para preservar a identidade das organizações participantes da pesquisa, seus nomes foram substituídos por letras do alfabeto em ordem crescente, de "A" a "P", correspondendo a cada uma das organizações. Esse procedimento foi adotado para preservar a identidade dos sujeitos pesquisados e garantir seu anonimato, conforme compromisso assumido com as organizações, a Tabela 1 apresenta a amostra das unidades pesquisadas.

Tabela 1 - Amostra das unidades pesquisadas.

\begin{tabular}{|c|c|c|c|}
\hline Organização & Área de atuação & Localização geográfica & Amostra \\
\hline A & Vestuário feminino & Norte & 29 \\
\hline B & Vestuário masculino e feminino & Oeste & 35 \\
\hline C & Vestuário masculino & Vale do Itajaí & 11 \\
\hline D & Vestuário masculino e feminino & Vale do Itajaí & 33 \\
\hline E & Vestuário masculino e feminino & Vale do Itajaí & 16 \\
\hline F & Vestuário masculino e feminino & Vale do Itajaí & 39 \\
\hline G & Vestuário masculino e feminino & Vale do Itajaí & 16 \\
\hline H & Produtos médico-hospitalares & Vale do Itajaí & 25 \\
\hline I & Cama, mesa e banho & Vale do Itajaí & 36 \\
\hline J & Vestuário masculino e feminino & Oeste & 30 \\
\hline K & Indústria de etiquetas & Vale do Itajaí & 27 \\
\hline L & Material esportivo & Oeste & 21 \\
\hline M & Material esportivo & Norte & 65 \\
\hline N & Produção de vestuário & Norte & 18 \\
\hline O & Vestuário infantil & Vale do Itajaí & 21 \\
\hline P & Vestuário masculino e feminino & Norte & 19 \\
\hline Total & & - & $\mathbf{4 4 1}$ \\
\hline
\end{tabular}

Fonte: Dados da pesquisa.

Conforme a Tabela 1 verifica-se que o maior número de organizações da amostra atua no setor de confecção, produzindo basicamente peças do vestuário pessoal (feminino, masculino e infantil) e doméstico (cama, mesa e banho). Uma característica do segmento de confecções é que ele demanda flexibilidade produtiva para o ajuste das organizações às novas tendências de moda. A Tabela 2 apresenta o porte das organizações pesquisadas conforme o critério de classificação do SEBRAE.

Tabela 2 - Porte das organizações pesquisadas.

\begin{tabular}{|l|c|c|c|c|}
\hline \multirow{2}{*}{ Porte da organização } & \multicolumn{2}{c|}{ Organizações participantes } & \multicolumn{2}{c|}{ Número de respondentes } \\
\cline { 2 - 5 } & Frequência & Percentual & Frequência & Percentual \\
\hline Organização de Pequeno Porte & 2 & 12,4 & 86 & 19,5 \\
\hline Organização de Médio Porte & 7 & 43,8 & 243 & 55,1 \\
\hline Organização de Grande Porte & 7 & 43,8 & 112 & 25,4 \\
\hline Total & $\mathbf{1 6}$ & $\mathbf{1 0 0}$ & $\mathbf{4 4 1}$ & $\mathbf{1 0 0}$ \\
\hline
\end{tabular}

Fonte: Dados da pesquisa.

As organizações têxteis foram divididas em pequenas, médias e grandes, conforme o número de empregados - segundo critérios do SEBRAE. Para operacionalizar essa variável e inseri-la como 
variável métrica no modelo, utilizou-se da reespecificação de variáveis por meio de variáveis dummy, ou seja, aquelas que podem assumir apenas dois valores. A reespecificação de variáveis é a transformação de dados para criar novas variáveis ou modificar variáveis existentes de modo a tornálas mais consistentes com o objetivo do estudo. As variáveis dummy compreendem um importante processo de reespecificação (Hair, Jr. et al., 2005). Assim, os indicadores foram transformados em variáveis dummy: um para grande empresa e zero para pequena e média empresa.

\section{APRESENTAÇÃO E ANÁLISE DOS RESULTADOS}

Este tópico mostra os resultados empíricos obtidos pela aplicação do instrumento de coleta de dados do desempenho em inovação de produtos na indústria têxtil de Santa Catarina. Foram procedidos cálculos destinados à validação das escalas propostas. Os dados obtidos por meio da aplicação dos questionários foram digitados em planilha Microsoft Excel ${ }^{\circledR}$ importadas pelo software SPSS® V.19, sendo gerados os índices de confiabilidade.

A validação da escala seguiu as recomendações científicas e se baseou na revisão de literatura e no estudo empírico. Inicialmente procedeu-se a análise fatorial exploratória que serviu para averiguar se cada variável dos constructos se relaciona somente com um fator, e se elas estão bem correlacionadas entre si, indicando um único fator, bem como a avaliação da unidimensionalidade dos constructos. Os indicadores passaram por uma série de testes para verificar se era possível criar agrupamentos que pudessem resultar em outros fatores, os quais, isolados dos identificados inicialmente, comporiam o modelo de avaliação de desempenho em inovação de produtos. Com base neste procedimento não foram excluídos indicadores, já que todos apresentaram resultados significativos para confiabilidade, matriz de correlação, carga fatorial e Comunalidades.

Após estas análises iniciais, procedeu-se a análise fatorial confirmatória (AFC) através do software AMOS ${ }^{\circledR} 20$, com a finalidade de verificar a validade dos constructos envolvidos no modelo de mensuração. Dado o conhecimento sobre as relações entre os constructos, procedeu-se à validação individual de cada um, de acordo com a análise de adequação dos índices de ajuste e a busca de evidências de validade convergente.

Os indicadores de confiabilidade - Confiabilidade Composta - CC, Variância Média Extraída AVE e Alfa de Cronbach - AF, bem como as medidas de ajustamento absoluto, incremental e parcimonioso, que mostraram o grau em que os indicadores especificados representam os constructos teorizados são apresentadas na Tabela 3.

Revista de Administração e Inovação, São Paulo, v. 11, n.2, p. 273-294, abr./jun. 2014. 
Tabela 3 - Índices de ajuste individuais para as dimensões de primeira ordem do desempenho em inovação de produtos.

\begin{tabular}{|l|c|c|c|c|c|}
\hline $\begin{array}{c}\text { Medidas de } \\
\text { ajuste }\end{array}$ & Nível aceitável & $\begin{array}{c}\text { Encontrado - } \\
\text { Eficácia }\end{array}$ & $\begin{array}{c}\text { Encontrado - } \\
\text { Eficiência }\end{array}$ & $\begin{array}{c}\text { Encontrado - } \\
\text { Melhorias }\end{array}$ & $\begin{array}{c}\text { Encontrado - } \\
\text { Custos }\end{array}$ \\
\hline GL & - & 18 & 2 & 2 & 9 \\
\hline$\chi 2 \mathrm{e} p$ & $-(\mathrm{p}<0,000)$ & $\begin{array}{c}87,597- \\
\mathrm{p}<0,000)\end{array}$ & $\begin{array}{c}6,958- \\
(\mathrm{p}<0,000)\end{array}$ & $\begin{array}{c}6,634- \\
(\mathrm{p}<0,000)\end{array}$ & $\begin{array}{c}17,672- \\
(\mathrm{p}<0,000)\end{array}$ \\
\hline$\chi 2 / \mathrm{GL}$ & $\leq 5$ & 4,867 & 3,479 & 3,317 & 1,964 \\
\hline GFI & $>0,90$ & 0,945 & 0,992 & 0,993 & 0,987 \\
\hline AGFI & $>0,90$ & 0,915 & 0,962 & 0,963 & 0,970 \\
\hline SRMR & $<0,10$ & 0,044 & 0,013 & 0,017 & 0,016 \\
\hline RMSEA & $0,05 \mathrm{a} 0,08$ & 0,094 & 0,075 & 0,073 & 0,047 \\
\hline TLI & $>0,90$ & 0,939 & 0,985 & 0,981 & 0,990 \\
\hline CFI & $>0,90$ & 0,948 & 0,995 & 0,994 & 0,994 \\
\hline PNFI & $>0 \mathrm{e}<1$, próximo de 1 & 0,802 & 0,331 & 0,330 & 0,593 \\
\hline \multicolumn{1}{|c|}{ AF } & $>0,70$ & 0,876 & 0,890 & 0,903 & 0,847 \\
\hline CC & $>0,70$ & 0,927 & 0,891 & 0,678 & 0,914 \\
\hline AVE & $>0,50$ & 0,514 & 0,670 & 0,350 & 0,653 \\
\hline
\end{tabular}

Fonte: Dados da pesquisa.

Analisando os índices de ajuste individuais para as dimensões de primeira ordem do desempenho em inovação de produtos (Tabela 3), contata-se que as medidas de ajuste absoluto, medidas de ajuste incremental e medidas de ajuste parcimonioso das dimensões de primeira ordem do modelo do desempenho em inovação de produtos ficaram dentro dos níveis aceitáveis. Observa-se que o teste de $\chi^{2}$ e RMSEA apresentam valores acima do nível aceitável. Entretanto as demais medidas de ajuste apresentam valores aceitáveis: GFI superior a 0,9. As três medidas de ajuste comparativas (CFI e TLI) apresentam valores acima de 0,9 , indicando que o modelo proposto é ajustado.

No que tange aos indicadores de confiabilidade, apenas a dimensão Melhorias apresentou índices abaixo do recomendado para confiabilidade composta - CC e para a variância média extraída - AVE, contudo o Alfa de Cronbach (AF) ficou acima do recomendado pela literatura. Para as demais dimensões todos apresentaram valores acima do sugerido pela literatura (Garver \& Mentzer, 1999, Hair, Jr. et al., 2005). As medidas de ajuste do modelo completo de segunda ordem são apresentadas na Tabela 4.

Tabela 4 - Índices de ajuste para o modelo de segunda ordem do desempenho em inovação de produtos. 


\begin{tabular}{|l|c|c|}
\hline \multicolumn{1}{|c|}{ Medidas de Ajuste } & Nível Aceitável & Nível encontrado - Final \\
\hline GL & - & 221 \\
\hline$\chi^{2} \mathrm{e} \mathrm{p}$ & $-(\mathrm{p}<0,000)$ & $666,182-(\mathrm{p}<0,000)$ \\
\hline$\chi^{2} / \mathrm{GL}$ & $\leq 5$ & 3,014 \\
\hline GFI & $>0,90$ & 0,877 \\
\hline AGFI & $>0,90$ & 0,859 \\
\hline SRMR & $<0,10$ & 0,057 \\
\hline RMSEA & $0,05 \mathrm{a} 0,08$ & 0,068 \\
\hline TLI & $>0,90$ & 0,921 \\
\hline CFI & $>0,90$ & 0,924 \\
\hline PNFI & $>0$ e $<1$, próximo de 1 & 0,857 \\
\hline
\end{tabular}

Fonte: Dados da pesquisa.

As medidas de ajuste absoluto, GFI e AGFI, embora próximas do valor aceitável de 0,90, não resultaram em valores satisfatórios. Entretanto é oportuno destacar que Bagozzi e Yi (2012) ressaltam que não existem critérios de corte para o GFI e AGFI, esses índices são dependentes do tamanho da amostra, e simulações mostram que ambos não apresentam resultados tão significativos como o RMSEA, o TLI, o CFI, e o SRMR. Assim, os autores apontam que para um bom ajustamento devem ser utilizados os indicadores: $\chi^{2}$, o RMSEA, o CFI, o TLI e o SRMR. Dentro dessas condições, o modelo geral foi considerado aceitável.

Quando analisada a qualidade do modelo, sob o indicador $\chi^{2}$ sobre GL, têm-se 3,014, o que comprova a sua qualidade, sendo estatisticamente significantes no nível de $0,000(p<0,05)$ (Hair, Jr. et al., 2005; Kline, 2005). O Root Mean Square Error of Aproximation - RMSEA apresentou o valor de 0,068, o Comparative Fit Index - CFI foi igual a 0,924, o Tucker-Lewis Index - TLI apresentou valor de 0,924 e o Standardized Root Mean-Square Residual - SRMR foi de 0,057. A Figura 1 apresenta o constructo final de segunda ordem do desempenho em inovação de produtos. 


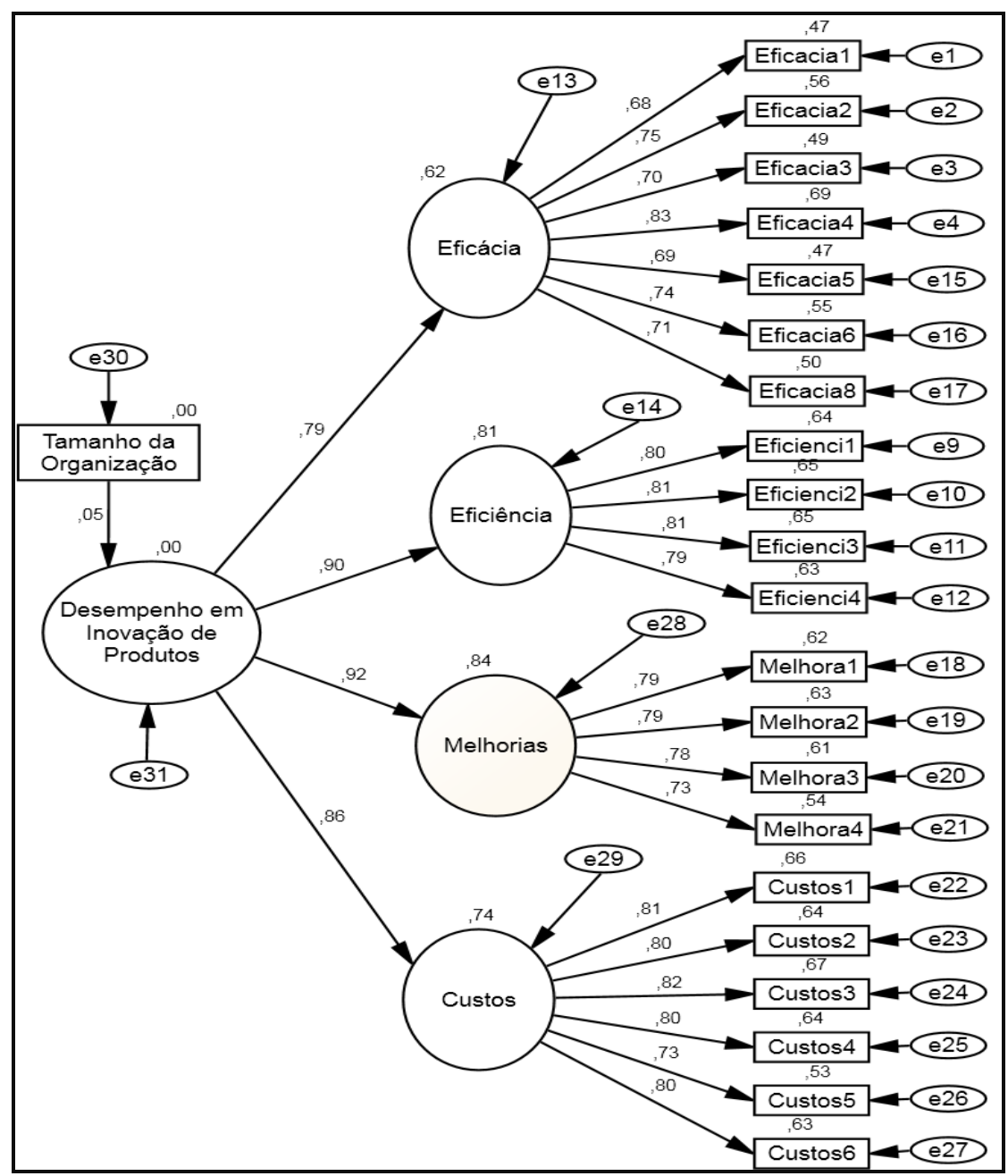

Figura 1 - Constructo final de segunda ordem do desempenho em inovação de produtos. Fonte: Dados da pesquisa.

Com base nas cargas fatoriais padronizadas, averiguou-se a validade das relações propostas no constructo de segunda ordem do desempenho em inovação de produtos. Conforme a Figura 1, o caminho (path) para dimensão de primeira ordem - Eficácia foi de $\lambda=0,79$ e o valor do coeficiente de determinação da variância $\left(\mathrm{R}^{2}\right)$ foi de $62 \%$. A dimensão de primeira ordem - Eficiência apresentou um coeficiente padronizado de $\lambda=0,90$, para um $\mathrm{R}^{2}$ de $81 \%$. Para a dimensão de primeira ordem Melhorias, o caminho (path) foi de $\lambda=0,92$ com $\mathrm{R}^{2}=84 \%$. E para a dimensão de primeira ordem Custos o caminho (path) foi de $\lambda=0,86$ e o coeficiente de determinação $\left(\mathrm{R}^{2}\right)$ foi de $74 \%$ da variância explicada.

Com o intuito de avaliar a influência do tamanho da organização no desempenho em inovação de produtos, foi incluída na regressão uma dummy para essa característica. Porém ela não foi significativa, com coeficiente de 0,005 e significância de 0,307, mostrando que o tamanho da organização não tem influência no desempenho em inovação de produtos na amostra das organizações pesquisadas. 
As cargas dos itens de medida sobre os fatores de primeira ordem e as cargas dos fatores de primeira ordem sobre os fatores de segunda ordem foram significativas para p, 0,001. Desse modo, as quatro dimensões formam o constructo de segunda ordem - desempenho em inovação de produtos. De modo geral, o constructo por meio das dimensões de primeira ordem - Eficácia, Eficiência, Melhorias e Custos - pode ser considerado um constructo de segunda ordem para a avaliação do desempenho em inovação de produtos da indústria têxtil. Os resultados estatísticos evidenciaram que os 21 itens convergem em um constructo único de segunda ordem.

\subsection{Teste e discussão das hipóteses do estudo}

Despois de calculados os índices de ajustamento do modelo final e obtidos os resultados por meio da MEE, as hipóteses são comentadas, assim como sua validação ou refutação a partir dos resultados obtidos na pesquisa de campo. A Tabela 5 apresenta o resumo dos testes de hipóteses.

Tabela 5 - Resumo dos testes de hipóteses.

\begin{tabular}{|c|l|c|c|c|c|c|}
\hline Hipótese & \multicolumn{1}{|c|}{ Caminho estrutural ou interação } & $\begin{array}{c}\text { Coeficiente } \\
\text { padronizado }\end{array}$ & $\begin{array}{c}\boldsymbol{t} \text { - } \\
\text { value }\end{array}$ & $\mathbf{R}^{\mathbf{2}}$ & Sig. & Resultado \\
\hline H1 & Eficácia $\leftarrow$ Desempenho em inovação de produtos & $0,791(1)$ & - & 0,625 & 0,000 & Aceita \\
\hline H2 & Eficiência $\leftarrow$ Desempenho em inovação de produtos & 0,901 & 14,535 & 0,812 & 0,000 & Aceita \\
\hline H3 & Melhorias $\leftarrow$ Desempenho em inovação de produtos & 0,914 & 13,671 & 0,836 & 0,000 & Aceita \\
\hline H4 & Custos $\leftarrow$ Desempenho em inovação de produtos & 0,861 & 14,120 & 0,742 & 0,000 & Aceita \\
\hline H5 & Desempenho em inovação de produtos $\leftarrow$ Tamanho & 0,052 & 1,021 & 0,000 & 0,307 & Rejeitada \\
\hline
\end{tabular}

(1) Valores iniciais fixados em 1,00.

Fonte: Elaboração própria.

A Tabela 5 apresentou os testes das hipóteses do estudo com o caminho estrutural, os coeficientes padronizados estimados, os t-values, o nível de significância, o status da hipótese na relação esperada. Na sequência são apresentadas e discutidas as hipóteses do estudo. A primeira hipótese previamente formulada apresentou a seguinte afirmação: Hipótese 1: A Eficácia tem um efeito positivo no desempenho em inovação de produtos na indústria têxtil de Santa Catarina.

Com base nos resultados da pesquisa de campo, a hipótese pode ser confirmada. Constatou-se que as organizações têxteis pesquisadas apresentaram uma correlação positiva entre a dimensão Eficácia e o desempenho em inovação de produtos, com um coeficiente beta padronizado de $\lambda=0,79$. Entre esses indicadores se sobressaem o desenvolvimento de novas linhas de produtos, o aumento na participação de mercado, a abertura de novos mercados nacionais e a ampliação da linha de produtos. Os resultados indicam que as organizações buscam a perpetuação no mercado, e é por meio de inovações em produtos para atender a demandas externas de um mercado que busca novos produtos

Revista de Administração e Inovação, São Paulo, v. 11, n.2, p. 273-294, abr./jun. 2014. 
que elas se inserem, corroborando estudos apresentados por Montoya-Weiss e Calantone (1994), Atuanaheme-Gima (1995), Barczak (1995) e Valle e Avella (2003).

A segunda hipótese formulada apresentou a seguinte afirmação: Hipótese 2: A Eficiência tem um efeito positivo no desempenho em inovação de produtos na indústria têxtil de Santa Catarina. Tendo em vista os resultados da pesquisa de campo, a hipótese pode ser confirmada. A dimensão Eficiência, ou seja, aquela que foca o processo de inovação que é executado nas fronteiras internas da organização, apresentou correlação forte e positiva com o desempenho em inovação de produtos na indústria têxtil catarinense, com um coeficiente beta padronizado de $\lambda=0,90$. As organizações estão preocupadas com o custo e o tempo do projeto despendido com o desenvolvimento da inovação (Griffin \& Page, 1993; Griffin, 1997; Zhan \& Doll, 2001; Mcevily \& Chakravarthy, 2002; Valle \& Avella, 2003). Griffin (1997) observa que o uso de equipes multifuncionais é uma forma de reduzir o tempo de ciclo de desenvolvimento. Isso ficou evidente no estudo, uma vez que o indicador sobre equipes multifuncionais teve alta correlação na dimensão Estrutura.

A terceira hipótese formulada apresentou a seguinte afirmação: Hipótese 3: As melhorias no processo de inovação têm um efeito positivo no desempenho em inovação de produtos na indústria têxtil de Santa Catarina. Com base nos resultados da pesquisa de campo, a hipótese pode ser confirmada. As melhorias no processo de inovação apresentaram correlação positiva com o desempenho em inovação de produtos na indústria têxtil catarinense, com um coeficiente beta padronizado de $\lambda=0,90$. Para Costa e Rocha (2009) é necessário que as organizações do setor têxtil procurem melhoria da qualidade do produto, substituir produtos velhos, diversificar a gama de produtos oferecidos e melhorar constantemente o desempenho dos mesmos. Para isso devem desenvolver novas tecnologias ou melhorar e adaptar, de maneira criativa, tecnologias existentes às necessidades de produção da empresa.

Evidencia-se que se deve inovar também nos processos, para que sejam mais competitivos, no aumento da qualidade, na melhoria das condições de trabalho, e na melhoria da flexibilidade produtiva. Para Naranjo-Valencia, Jiménez, Sanz-Valle (2012) fatores associados a uma maior flexibilidade, fundamentais para alcançar a inovação - como criatividade, autonomia e propensão ao risco -, são difíceis de ser maximizados quando a organização coloca ênfase na estabilidade e no controle.

A quarta hipótese formulada expressa a seguinte afirmação: Hipótese 4: A redução de custos no processo de inovação tem um efeito positivo no desempenho em inovação de produtos na indústria têxtil de Santa Catarina. A Hipótese 4 pode ser confirmada conforme os resultados da pesquisa de campo. Os custos no processo de inovação apresentaram correlação positiva com o desempenho em

Revista de Administração e Inovação, São Paulo, v. 11, n.2, p. 273-294, abr./jun. 2014. 
inovação de produtos na indústria têxtil catarinense, com um coeficiente beta padronizado de $\lambda=0,86$. A flexibilização da produção e do trabalho são geralmente estratégias adotadas pelas organizações para a obtenção de menores custos de produção. Os custos de produção podem ser reduzidos por meio de ganhos de escala e inovação com processos conjuntos de P\&D. (Rech, 2006; OCDE, 2005; Freeman \& Soete, 2008).

As organizações da indústria têxtil necessitam reduzir os custos de produção para tornar os produtos mais competitivos, tendo em vista o crescimento dos produtos asiáticos nos mercados mundiais, em especial os da China, que desestabilizou os demais países produtores de têxteis e confeccionados acirando a competição global. Nessa conjuntura, para que as organizações do setor se mantenham competitivas, é fundamental a redução dos custos de produção por meio de cortes no consumo de materiais, energia, redução da taxa de produção de rejeitados entre outros (Costa, \& Rocha, 2009).

A última hipótese do estudo foi formulada com afirmação: Hipótese 5: $O$ tamanho da organização - conforme critérios pelo número de empregados - têm efeito positivo no desempenho em inovação de produtos na indústria têxtil de Santa Catarina. De acordo com os resultados, foi possível verificar que o tamanho da organização não teve impacto significativo no desempenho em inovação de produtos na indústria têxtil de Santa Catarina. As tecnologias da informação minimizam as perdas de tecido e os defeitos decorrentes do processo de produção, fazendo com que as organizações de pequeno e grande porte possam competir em igualdade (Rangel, Silva, \& Costa, 2010).

Os resultados foram contrários aos estudos de Damanpour e Evan (1984), Kimberly e Evanisko (1981) e Damanpour (1991), que revelaram que o porte da organização influencia a adoção de mais inovações. Os autores salientam que organizações maiores tendem a adotar mais inovações que organizações menores. Esse fato pode ser atribuído à disponibilidade de recursos: organizações maiores possuem mais condições econômicas e de capital intelectual para investir em novas ideias, enquanto organizações menores possuem seus recursos financeiros e de capital intelectual limitados, com maior dificuldade em disponibilizá-los para a inovação.

Nesse sentido, o porte da organização poderá afetar indiretamente a inovação, uma vez que tende a promover o aumento da centralização. $O$ setor têxtil brasileiro é majoritariamente formado por pequenas e médias empresas, apresentando elevado grau de concorrência e baixos índices de concentração industrial (Costa, \& Rocha, 2009).

Revista de Administração e Inovação, São Paulo, v. 11, n.2, p. 273-294, abr./jun. 2014. 


\section{CONCLUSÃO}

Com base nos resultados da pesquisa de campo, verificou-se que o desempenho na inovação de produtos pode ser abordado utilizando as dimensões de primeira ordem eficácia, eficiência, melhorias no processo de produção e redução de custos. Os resultados foram maiores na dimensão melhorias, refletindo maior atenção no aprimoramento da qualidade do produto, nas condições de trabalho e na flexibilidade produtiva.

Percebeu-se também uma preocupação com o custo e com o tempo gasto no desenvolvimento dos projetos de inovação. Por ser um setor da indústria que está sendo ameaçado pela constante competição dos chamados "tigres asiáticos", supõe-se que o foco esteja em uma competição por baixos custos de produção. Outro fator que pode ser apontado é a rápida variação das tendências de moda, o que leva as organizações desse setor a desenvolverem produtos que permanecem no mercado por curtos períodos de tempo.

No desempenho em inovação de produtos, a Eficiência ainda é mais importante que a Eficácia, indicando que o foco no processo de inovação é mais interno do que externo. Esse resultado pode ser atribuído à busca por baixar os custos na competitividade com os produtos provenientes da Ásia, sobretudo da China, que produzem com custos muito menores que o mercado nacional.

A concorrência asiática pressiona o setor têxtil brasileiro em todas as fases, da indústria de tecidos à de confecções, exigindo uma maior preocupação com a diminuição no tempo médio e no custo do desenvolvimento dos projetos de inovação. Entretanto, cabe salientar que, com a diminuição dos custos de produção, as margens de lucro tendem a diminuir consideravelmente, e apenas as organizações que atuam em economias de grande escala podem sobreviver. Nesse sentido, fica evidente que, com um processo bem gerenciado, voltado para a inovação, as organizações do setor podem desenvolver produtos de alto valor agregado, fazendo frente aos produtos importados.

O tamanho da organização não teve efeito significativo no desempenho em inovação de produtos nas organizações estudadas. O porte da organização pode afetar indiretamente a inovação, uma vez que tende a promover o aumento da centralização, porém isso não se confirmou nesta pesquisa. As pequenas e médias organizações conseguem competir com as grandes organizações investindo em tecnologia da informação, a qual possibilita diminuir as perdas decorrentes do processo de produção. Com esses progressos, pequenas confecções com máquinas de costura convencionais conseguem ser competitivas.

Entende-se que os resultados alcançados pelo presente estudo possam ser considerados relevantes tanto por seu impacto sobre a teoria quanto por sua aplicação gerencial. Pesquisas futuras devem ser direcionadas no sentido de analisar se em outros setores existem diferenças nos constructos

Revista de Administração e Inovação, São Paulo, v. 11, n.2, p. 273-294, abr./jun. 2014. 
do desempenho em inovação de produtos em decorrência de características particulares de cada setor. Outra iniciativa de pesquisa seria incrementar na escala de desempenho em inovação de produtos outras medidas objetivas, como patentes ou elevação na quantidade da produção.

\section{REFERÊNCIAS}

Afuah, A. (2003). Innovation mangement: strategies, implementation and profits. 2 ed. New York: Oxford University Press.

Alegre, J.; Lapiedra, R.; Chiva, R. (2006). A measurement scale for product innovation performance. European Journal of Innovation Management, v. 9, n. 4, pp. 333-346.

Alegre, J.; Chiva, R. (2008). Assessing the impact of organizational learning capability on product innovation performance: an empirical test. Technovation, v. 28, n. 1, pp. 315-326.

Alegre, J.; Chiva, R.; Lapiedra, R. (2009). Measuring innovation in long product development cycle industries: an insight in biotechnology. Technology Analysis \& Strategic Management, v. 21, n. 4, pp. $535-546$.

Ancona, D. G.; Caldwell, D.F. (1992). Bridging the boundary: external process and performance in organizational teams. Administrative Science Quaterly, v. 37, n. 4, pp. 634-665.

Atuanaheme-Gima, K. (1995). An exploratory study of the impact of market orientation on new product performance: a contingency approach. Journal of Product Innovation Management, v. 12, $\mathrm{n}$. 4, pp. 275-93.

Bagozzi, R. P.; Phillips, L. W. (1982). Representing and testing organizational theories: a holistic construal. Administrative Science Quarterly, v. 27, n. 3, pp. 459-489.

Bakar, L. J. A.; Ahmad, H. (2010). Assessing the relationship between firm resources and product innovation performance: A resource-based view. Business Process Management Journal, v. 16, n. 3, pp. 420-435.

Barczak, G. (1995). New product strategy, structure, process, and performance in the telecommunications industry. Journal of Product Innovation Management, v. 12, n. 3, pp. 224-34.

Chiesa, V.; Coughlan, P.; Voss, C.A. (1996). Development of a technical innovation audit. R\&D Management, v. 13, pp. 105-136.

Coombs, R.; Tomlinson, M. (1998). Patterns in UK company innovation styles: new evidence from the CBI innovation trends survey. Technology Analysis and Strategic Management, v. 12, n. 3, pp. 295310 .

Cooper, D. R.; Schindler, P. S. (2003). Métodos de pesquisa em administração. 7. ed. Porto Alegre: Bookman.

Revista de Administração e Inovação, São Paulo, v. 11, n.2, p. 273-294, abr./jun. 2014. 
Costa, A. C. R.; Rocha, E. R. P.. (2009). Panorama da cadeia produtiva têxtil e de confecções e a questão da inovação. BNDES Setorial, Rio de Janeiro, n. 29, pp. 159-202, mar.

Damanpour, F. (1996). Organizational complexity and innovation: developing and testing multiple contingency models. Management Science, New Jersey, v. 42, n. 5, pp. 693.

Damanpour, F. (1991). Organizational Innovation: A meta-analysis of effects of determinants and moderators. Academy of Management Journal. v. 34, n.3, p. 555-590.

Damanpour, F.; Evan, W. M. (1984). Organizational innovation and performance: the problem of 'organizational lag'. Administrative Science Quarterly, n. 29, pp. 392-409.

Ettlie, J. E. (1983). Organizational policy and innovation among suppliers to the food processing sector. Academy of Management Journal, v. 26, n.1, pp. 27-44.

Fernandes, R.; Cario, S.. (2009). Desempenho comercial e padrão de concorrência internacional: uma análise do setor têxtil-confecções catarinense entre 1996 e 2006. Indicadores Econômicos FEE, v. 36, n. 4, pp. 1-18.

Freeman C.; Soete, L.. (2008). A Economia da Inovação Industrial. Campinas: Editora Unicamp. Coleção Clássicos da Inovação.

Garver, N. S.; Mentzer, J. T. (1999). Logistics research methods: employing structural equation modeling to test for construct validity. Journal of Business Logistics, v. 20, n. 1, pp. 33-57.

Griffin, A. (1997). PDMA research on new product development practices: updating trends and benchmarking best practices. Journal of Product Innovation Management, v. 14, n. 6, pp. 429-459.

Griffin, A.; Page, A. (1993). An interim report on measuring product development success and failure. Journal of Product Innovation Management, v. 10, pp. 291-308.

Gupta, A. K.; Wilemon, D.L. (1990). Accelerating the development of technology-based new products. California Management Review, v. 32, n. 2, pp. 24-44.

Hair Jr., J. F.; Babin, B.; Money, A. H.; Samouel, P. (2005). Fundamentos e métodos de pesquisa em administração. Porto Alegre: Bookman.

Henttonen, K.; Ritala, P.; Jauhiainen, T. (2011). Exploring open search strategies and their perceived impact on innovation performance - empirical evidence. International Journal of Innovation Management, v. 15, n. 3, pp. 525-541.

Hoopes, D.G.; Postrel, S. (1999). Shared knowledge, 'glitches', and product development performance. Strategic Management Journal, v. 20, n. 9, p. 837-968.

Hull, F. M. Hage, J. Azumi K. (1985). R\&D Management strategies: Americam versus Japan. IEEE. Transactions on Enginnering Manegement, v. 32, n.4, pp. 78-83.

Jonash, R. S.; Sommerlatte, T. (2001). O valor da inovação: como as empresas mais avançadas atingem alto desempenho e lucratividade. Rio de Janeiro: Campus.

Revista de Administração e Inovação, São Paulo, v. 11, n.2, p. 273-294, abr./jun. 2014. 
Kimberly, J. R.; Evanisko, M. J. (1981). Organizational innovation: the influence of individual organizational, and contextual factors on hospital adoption of technological and administrative innovations. Academy of Management Journal, v. 24, n. 4, pp. 689- 713.

Kleinknecht, A. (1993). Why do we need new innovation output indicators?, In.: A. Kleinknecht; D. Bain (ed.): New concepts in innovation output measurement. New York: St. Martin’s Press.

Kline, R. B. (2005). Principles and practice of structural equation modeling. New York, The Guilford Press.

Knight, K. E. (1967). A descriptive model of intra-firm innovation process. Journal of Business, n. 40, pp. 478-496.

Mabert, V.A., Muth, J.F.; Schmenner, R.W. (1992). Collpasing new product development times: six case studies. Journal of Product Innovation Management, v. 9, pp. 200-212.

Mcevily, S.; Chakravarthy, B. (2002). The persistence of knowledge-based advantage: an empirical test for product performance and technological knowledge. Strategic Management Journal, v. 23, pp. 285-305.

Mendes, S. F.. (2003). Filiais brasileiras na rede mundial do setor têxtil: análise de algumas empresas industriais globalizadas a partir da gama de produtos, das etapas produtivas e das funções corporativas. Relatório de atividades final CNPq. Araraquara.

Montoya-Weiss, M.M.; Calantone, R.J. (1994). Determinants of new product performance: a review and meta-analysis. Journal of Product Innovation Management, v. 11, n. 5, pp. 397-417.

Naranjo-Valencia, J. C.; Jiménez, D. J.; Sanz-Valle, R. (2012). ¿Es la cultura organizativa un determinante de la innovación en la empresa? Cuadernos de Economía y Dirección de la Empresa, $\mathrm{v}$. 15, n. 3, pp.63-72.

OCDE - Organização para a Cooperação e o Desenvolvimento. (2005). Manual de Oslo. 3ed. Finep tradução português.

Oecd - Eurostat. (1997). The measurement of scientific and technological activities. Proposed guidelines for collecting and interpreting technological data. In: Oslo Manual. OECD, Paris,.

Pisano, G.P. (1994). Knowledge, integration and the locus of learning: an empirical analysis of process development. Strategic Management Journal, Winter Special Issue, v. 15, pp.85-100.

Rangel, A. S.; Silva, M. M.; Costa, B. K.. (2010). Competitividade da indústria têxtil brasileira. Revista de Administração e Inovação, São Paulo, v. 7, n. 1, p. 151-174, jan./mar.

Rech, S. R.. (2006). 282 f. Cadeia produtiva da moda: um modelo conceitual de análise da competitividade no elo confecção. Tese (Doutorado em Engenharia de Produção) - Universidade Federal de Santa Catarina - Programa de Pós-Graduação em Engenharia de Produção.

Sebrae - Serviço Brasileiro de Apoio a Micro e Pequenas Empresas. (2012). Site do SEBRAE. Disponível em <http:// sebrae.com.br>. Acesso em 20 de julho de 2012. 
Sebrae - Serviço Brasileiro de Apoio a Micro e Pequenas Empresas. (2012). Santa Catarina em números: têxtil e confecção. Disponível em: <http://www.sebraesc.com.br/scemnumero/arquivo/Texti-e-Confeccao.pdf>. Acesso em: 18 de março de 2012.

Tidd, J.; Bessant, J.; Pavitt, K. (2005). Managing Innovation: Integrating Technological, Market and Organizational Change. John Wiley \& Sons.

Utterback, J. M., Albernathy, W. J. (1975). A dynamic modelo of process and reorientation. Administrative Science Quarterly, n. 16, pp. 203-215.

Valle, S.; Avella, L. (2003). Cross-functionality and leadership of the new product development teams. European Journal of Innovation Management, v. 6, n. 1, pp. 32-47.

Van De Ven, A. H.; Polley, D. E.; Garud, R.; Venkataraman, S.. (1999). The Innovation Journey, Oxford University Press; New York.

Wheelwright, S.C.; Clark, K.B. (1992). Revolutionizing Product Development - Quantum Leaps in Speed, Efficiency, and Quality, The Free Press, New York.

Zhan, Q.; Doll, W.J. (2001). The fuzzy front end and succes of new product development causal model, European Journal of Innovation Management, v. 4, n. 2, pp. 95-112.

Zhuang, L.; Wiliamson, D.; Carter, M. (1999). Innovate or liquidate - are all organizations convinced? A two-phased study into the innovation process. Management Decision, v. 37, n. 1, pp. $57-71$. 


\title{
TEXTILE INDUSTRY OF SANTA CATARINA AND INNOVATIVE CAPACITY: STUDY IN THE PERSPECTIVE OF EFFICIENCY, EFFECTIVENESS, COST AND PROCESS IMPROVEMENT
}

\begin{abstract}
Santa Catarina stands as one of the major parks productive textile sector. Like other Brazilian textile industries, the Santa Catarina market comes under pressure, mainly from Asian countries. The study aimed to identify the dimensions - efficiency, effectiveness, cost and process improvements - are associated with the performance of innovation in the textile industry of Santa Catarina. The research was descriptive, exploratory and causal, survey or survey and quantitative. The sample consisted of 441 respondents from 16 organizations in the state of Santa Catarina. Data were analyzed using exploratory factor analysis, confirmatory factor analysis and structural equation modeling. The results show that the performance in product innovation can be addressed using the first-order dimensions effectiveness, efficiency improvements in the production process and reduce costs. The results were greater improvements in size, reflecting greater attention on the improvement of product quality, working conditions and production flexibility. The size of the organization had no significant effect on performance in product innovation in the organizations studied. It is understood that the results achieved by this study may be considered relevant both for their impact on the theory and its implementation by management. Future research should be conducted to examine whether there are differences in other sectors in the constructs of performance in product innovation due to particular characteristics of each sector.
\end{abstract}

Keywords: Innovative Capacity; Innovation in Products and Processes; Textile Industry.

Data do recebimento do artigo: 15/12/2013

Data do aceite de publicação: 25/05/2014

Revista de Administração e Inovação, São Paulo, v. 11, n.2, p. 273-294, abr./jun. 2014. 